\title{
OUTER FUNCTIONS IN FUNCTION ALGEBRAS ON THE BIDISC
}

\author{
HÅKAN HEDENMALM
}

\begin{abstract}
Let $f$ be a function in the bidisc algebra $A\left(\mathbf{D}^{2}\right)$ whose zero set $Z(f)$ is contained in $\{1\} \times \overline{\mathbf{D}}$. We show that the closure of the ideal generated by $f$ coincides with the ideal of functions vanishing on $Z(f)$ if and only if $f(\cdot, \alpha)$ is an outer function for all $\alpha \in \overline{\mathbf{D}}$, and $f(1, \cdot)$ either vanishes identically or is an outer function. Similar results are obtained for a few other function algebras on $\mathbf{D}^{2}$ as well.
\end{abstract}

0. Introduction. In 1926, Torsten Carleman [CAR; GRS, §45] proved the following theorem: $A$ function $f$ in the disc algebra $A(\mathrm{D})$, vanishing at the point 1 only, generates an ideal that is dense in the maximal ideal $\{g \in A(\mathbf{D}): g(1)=0\}$ if and only if

$$
\lim _{\mathbf{R} \ni t \rightarrow 1^{-}}(1-t) \log |f(t)|=0 .
$$

This condition, which Carleman refers to as $f$ having no logarithmic residue, is equivalent to $f$ being an outer function in the sense that

$$
\log |f(0)|=\frac{1}{2 \pi} \int_{-\pi}^{\pi} \log \left|f\left(e^{i \theta}\right)\right| d \theta .
$$

Carleman's result was an early predecessor of the Beurling-Rudin Theorem [RUD1; HOF, pp. 82-89], which completely describes the collection of all closed ideals in $A(\mathbf{D})$.

For a function $f$ in the bidisc algebra $A\left(\mathbf{D}^{2}\right)$, let $Z(f)=\left\{z \in \overline{\mathbf{D}}^{2}: f(z)=0\right\}$ be its zero set, and denote by $I(f)$ the closure of the principal ideal in $A\left(\mathbf{D}^{2}\right)$ generated by $f$. For $E \subset \overline{\mathbf{D}}^{2}$, introduce the notation $I(E)=\left\{f \in A\left(\mathbf{D}^{2}\right): f=0\right.$ on $E$ \}. The purpose of this paper is to try to obtain for $A\left(\mathbf{D}^{2}\right)$ results similar to Carleman's. More precisely, we consider the following problem.

Problem. Characterize those functions $f$ in $A\left(\mathbf{D}^{2}\right)$ with $Z(f) \subset \partial\left(\mathbf{D}^{2}\right)$ for which $I(f)=I(Z(f))$.

According to the Beurling-Rudin theorem, the answer to the same problem for the disc algebra $A(\mathbf{D})$ is that $f$ should be an outer function. One could say that we seek those functions of the bidisc algebra that are outer in the sense of the Beurling-Rudin theorem. (See [RUD2, pp. 70-83; RUS, ACD] for information on related questions.)

We will consider this problem for small zero sets $Z(f)$ only. For singleton $Z(f)$, the answer is surprisingly uncomplicated, as we shall see. First observe that the

Received by the editors February 15, 1987.

1980 Mathematics Subject (llassification (1985 Revision). Primary 32E25, 46J15, 46J20; Secondary $30 \mathrm{H} 05,42 \mathrm{~B} 10,43 \mathrm{~A} 10$.

This research was supported by the Swedish Natural Science Research Council (NFR). 
point in $Z(f)$ must lie in the Silov boundary $\mathbf{T}^{2}=(\partial \mathbf{D})^{2}$, so after a rotation we may assume $Z(f)=\{(1,1)\}$. This is so because if $f(\alpha)=0$ for some $\alpha=\left(\alpha_{1}, \alpha_{2}\right) \in \overline{\mathbf{D}}^{2}$ with $\left|\alpha_{1}\right|<1$, say, then, as $z_{2} \rightarrow \alpha_{2}$, the nonzero functions $f\left(\cdot, z_{2}\right)$ would converge to the function $f\left(\cdot, \alpha_{2}\right)$ that vanishes at the interior point $\alpha_{1}$ only, which is clearly impossible. The answer is as follows: $I(f)=I(\{(1,1)\})$ if and only if both $f(1, \cdot)$ and $f(\cdot, 1)$ are outer functions in $A(\mathbf{D})$. This is a consequence of the following more general result. For a function $f \in A\left(\mathbf{D}^{2}\right)$ with $Z(f) \subset\{1\} \times \overline{\mathbf{D}}$ to have $I(f) \supset I(\{1\} \times \overline{\mathbf{D}})$, it is necessary and sufficient that $f(\cdot, z)$ is outer for all $z \in \overline{\mathbf{D}}$. Equipped with this result, one can show, by invoking the Beurling-Rudin theorem, that $I(f)=I(Z(f))$ if and only if $f(\cdot, z)$ is outer for all $z \in \overline{\mathbf{D}}$, and $f(1, \cdot)$ either vanishes identically or is an outer function.

The above-mentioned results are proved in $\S 2$. Our main tool is a modification to a several complex variable setting of a well-known device in ideal theory for function algebras on planar domains, known as the Carleman transform (see [DOM, KOR2]). In $\S 3$, we obtain a similar result for the nonseparable algebra $H^{\infty}\left(\mathbf{D}^{2}\right)$. The main difficulty here is the complicated structure of the maximal ideal space.

The methods developed in this paper can also be applied to function algebras where the norm is not uniform. In $\S 4$, we study the algebra

$$
H_{1,1}^{2}\left(\mathbf{D}^{2}\right)=\left\{f \in H^{2}\left(\mathbf{D}^{2}\right): \partial f / \partial z_{1}, \partial f / \partial z_{2}, \partial^{2} f / \partial z_{1} \partial z_{2} \in H^{2}\left(\mathbf{D}^{2}\right)\right\},
$$

endowed with the natural norm. We may also think of this algebra as consisting of complex-valued sequences $\left\{a_{k n}\right\}_{k, n \in \mathbf{N}}$ satisfying

$$
\sum_{k, n}\left(1+k^{2}\right)\left(1+n^{2}\right)\left|a_{k n}\right|^{2}<\infty
$$

where the multiplication is sequence convolution. The function corresponding to a sequence $\left\{a_{k n}\right\}$ is its Taylor series $\left(z_{1}, z_{2}\right) \mapsto \sum_{k, n} a_{k n} z_{1}^{k} z_{2}^{n}, z_{1}, z_{2} \in \overline{\mathbf{D}}$. Let us introduce the same notation as for $A\left(\mathrm{D}^{2}\right)$; for instance, let $I(f)$ denote the closure (in $H_{1,1}^{2}\left(\mathbf{D}^{2}\right)$ ) of the principal ideal generated by $f \in H_{1,1}^{2}\left(\mathbf{D}^{2}\right)$. It turns out that a function $f \in H_{1,1}^{2}\left(\mathbf{D}^{2}\right)$ with $Z(f) \subset\{1\} \times \overline{\mathbf{D}}$ has $I(f) \supset I(\{1\} \times \overline{\mathbf{D}})$ if and only if $f(\cdot, z)$ is outer for all $z \in \overline{\mathbf{D}}$, just as for $A\left(\mathbf{D}^{2}\right)$. At this point we invoke a result by Korenblum [KOR1], describing the collection of closed ideals in the algebra $H_{1}^{2}(\mathbf{D})=\left\{f \in H^{2}(\mathbf{D}) ; f^{\prime} \in H^{2}(\mathbf{D})\right\}$ to show that $I(f)=I(Z(f))$ happens for an $f \in H_{1,1}^{2}\left(\mathbf{D}^{2}\right)$ with $Z(f) \subset\{1\} \times \overline{\mathbf{D}}$ if and only if $f(\cdot, z)$ is outer for all $z \in \overline{\mathbf{D}}$, and $f(1, \cdot)$ either vanishes identically or is an outer function.

The problems considered in this paper are intimately connected with an old open problem in harmonic analysis, raised by B. Ya. Levin, which recently appeared in [LEV]. He asks for a complete description of those functions $f \in L^{1}\left(\mathbf{R}_{+}^{2}\right)$, extended to vanish on $\mathbf{R}^{2} \backslash \mathbf{R}_{+}^{2}$, where $\mathbf{R}_{+}^{2}=\mathbf{R}_{+} \times \mathbf{R}_{+}$and $\mathbf{R}_{+}=(0, \infty)$, for which combined right and upper translates

$$
T_{x} f(t)=f(t-x), \quad t, x \in \mathbf{R}_{+}^{2},
$$

span a dense subspace of $L^{1}\left(\mathbf{R}_{+}^{2}\right)$. The one-dimensional version of this problem was solved by Bertil Nyman [NYM]. In $\S 5$, we solve a corresponding question for a convolution algebra of Borel measures supported on $\overline{\mathbf{R}}_{+}^{2}$ that are absolutely continuous with respect to area measure on $\mathbf{R}_{+}^{2}$. It is also explained that if we 
disregard the difference between the $L^{1}\left(\mathbf{R}_{+}^{2}\right)$ norm and the supremum norm of the Fourier transform, Levin's problem can be formulated as follows. Given a function $f \in A\left(\mathbf{D}^{2}\right)$ with $Z(f)=(\{1\} \times \overline{\mathbf{D}}) \cup(\overline{\mathbf{D}} \times\{1\})$, under what conditions on $f$ is $I(f)=I(Z(f))$ ? Here, the shape and size of $Z(f)$ is the source of the difficulty.

1. Preliminaries. If a function $f \in H^{\infty}(\mathbf{D})$ has no zeros on D, its Herglotz representation takes the form

$$
f(z)=\lambda \exp \left\{\int_{-\pi}^{\pi} \frac{e^{i \theta}+z}{e^{i \theta}-z}\left(\log \left|f\left(e^{i \theta}\right)\right| d \theta / 2 \pi-d \mu\left(e^{i \theta}\right)\right)\right\}, \quad z \in \mathbf{D},
$$

where $\mu$ is a nonnegative singular (Borel) measure on $\mathbf{T}=\partial \mathbf{D}$, and $\lambda \in \mathbf{C},|\lambda|=1$. It is easy to check that the limit

$$
\lim _{\mathbf{R} \ni t \rightarrow 1^{-}}(1-t) \log |f(t)|
$$

exists and equals $2 \mu(\{1\})$. The following lemma will prove useful.

LEMMA 1.1. Let $\left\{f_{n}\right\}_{0}^{\infty}$ be a bounded sequence of functions in $H^{\infty}(\mathbf{D})$ that are zero-free on $\mathbf{D}$, which converges uniformly on compact subsets of $\mathbf{D}$ to a function $f \in H^{\infty}(\mathbf{D})$, also zero-free on $\mathbf{D}$. If, for some sequence $\left\{t_{n}\right\}_{0}^{\infty} \subset[0,1), t_{n} \nearrow 1$,

$$
\lim _{n \rightarrow \infty}\left(1-t_{n}\right) \log \left|f_{n}\left(t_{n}\right)\right|=-\alpha \in[-\infty, 0],
$$

then

$$
\lim _{t \rightarrow 1^{-}}(1-t) \log |f(t)| \leq-\alpha
$$

In particular, $\alpha$ cannot be infinite.

PROOF. Without loss of generality, we can assume that $\left\|f_{n}\right\| \leq 1$. Look at the positive harmonic functions $h_{n}=-\log \left|f_{n}\right|$, which satisfy

$$
\left\|h_{n}\right\|_{h^{1}}=h_{n}(0) \leq C
$$

independently of $n$, because $h_{n}(0) \rightarrow-\log |f(0)|$ as $n \rightarrow \infty$. Denote by $\mu_{n}$ the nonnegative Borel measure on $\mathbf{T}$ for which

$$
h_{n}(z)=P_{z}\left[\mu_{n}\right]=\int_{-\pi}^{\pi} P_{z}\left(e^{i \theta}\right) d \mu_{n}\left(e^{i \theta}\right), \quad z \in \mathbf{D}
$$

where $P_{z}\left(e^{i \theta}\right)$ is the Poisson kernel $\left(1-|z|^{2}\right) /\left(\left|e^{i \theta}-z\right|^{2}\right)$. The space $h^{1}$ can be identified (by means of Poisson integrals) with $M(\mathbf{T})$, the space of all finite Borel measures on T [RUD3, p. 262], which in its turn is the dual space of $C(\mathbf{T})$. Since $C(\mathbf{T})$ is separable, the closed unit ball in $M(\mathbf{T})$ is compact and metrizable in the weak* topology. Hence we may extract a subsequence $\left\{\mu_{n_{k}}\right\}$ which converges, weak $^{*}$, to a $\mu \in M(\mathbf{T}), \mu \geq 0$, because $\left\|\mu_{n}\right\|=\left\|h_{n}\right\|_{h^{1}} \leq C$. Without loss of generality, we may replace the sequence $\left\{f_{n}\right\}$ by its subsequence $\left\{f_{n_{k}}\right\}$. Then

$$
\log 1 /\left|f_{n}(z)\right|=P_{z}\left[\mu_{n}\right] \rightarrow P_{z}[\mu]
$$

as $n \rightarrow \infty$, and hence $\log 1 /|f(z)|=P_{z}[\mu]$. The assertion of the lemma is that $\mu(\{1\}) \geq \alpha / 2$. Observe that for $t, 0 \leq t<1$,

$$
P_{t}\left(e^{i \theta}\right)=\frac{1-t^{2}}{\left|e^{i \theta}-t\right|^{2}} \leq \frac{2}{\left|e^{i \theta}-t\right|} \leq \frac{2}{1-t} .
$$


Fix an open arc $I \subset \mathbf{T}$ containing 1 . Then

$$
\sup _{0 \leq t<1} \int_{\mathbf{T} \backslash I} P_{t}\left(e^{i \theta}\right) d \mu_{n}\left(e^{i \theta}\right) \leq C(I)
$$

for some constant $C(I)$ independent of $n$, because $\left\|\mu_{n}\right\|=h_{n}(0) \leq C$ and

$$
\left\|P_{t}\right\|_{L^{\infty}(\mathbf{T} \backslash I)} \leq 2 / d(t, \mathbf{T} \backslash I),
$$

where $d$ is the Euclidean metric in $\mathbf{C}$, and so

$$
\begin{aligned}
\left(1-t_{n}\right) h_{n}\left(t_{n}\right) & =\left(1-t_{n}\right) \int_{\mathbf{T}} P_{t_{n}}\left(e^{i \theta}\right) d \mu_{n}\left(e^{i \theta}\right) \\
& =\left(1-t_{n}\right) \int_{\mathbf{T} \backslash I} P_{t_{n}}\left(e^{i \theta}\right) d \mu_{n}\left(e^{i \theta}\right)+\left(1-t_{n}\right) \int_{I} P_{t_{n}}\left(e^{i \theta}\right) d \mu_{n}\left(e^{i \theta}\right) \\
& \leq C(I) \cdot\left(1-t_{n}\right)+2 \mu_{n}(I) .
\end{aligned}
$$

Letting $n \rightarrow \infty$ and recalling that the left-hand side tends to $\alpha$, we conclude that

$$
\liminf _{n \rightarrow \infty} \mu_{n}(I) \geq \alpha / 2 .
$$

Let $\chi_{I, \varepsilon}$ be a continuous, real valued function on $\mathbf{T}$ such that $0 \leq \chi_{I, \varepsilon}(z) \leq 1$ on $\mathbf{T}, \chi_{I, \varepsilon}(z)=1$ on $I$, and $\chi_{I, \varepsilon}(z)=0$ outside an $\varepsilon$-neighborhood of $I$. Then

$$
\liminf _{n \rightarrow \infty}\left\langle\chi_{I, \varepsilon}, \mu_{n}\right\rangle \geq \alpha / 2,
$$

and since $\mu_{n} \rightarrow \mu$ weak $^{*}$, we conclude that $\left\langle\chi_{I, \varepsilon}, \mu\right\rangle \geq \alpha / 2$. Letting $\varepsilon \rightarrow 0$, it follows that $\mu(\bar{I}) \geq \alpha / 2$. Since the arc $I$ was arbitrary, we must have $\mu(\{1\}) \geq \alpha / 2$. This completes the proof.

Proposition 1.2. Let $f \in A\left(\mathbf{D}^{2}\right)$ have $Z(f) \subset\{1\} \times \overline{\mathbf{D}}$. Then the following two conditions are equivalent:

(a) $\lim _{t \rightarrow 1^{-}}(1-t) \log |f(t, z)|=0$ for all $z \in \overline{\mathbf{D}}$,

(b) $\lim _{t \rightarrow 1^{-}} \inf _{z \in \overline{\mathbf{D}}}(1-t) \log |f(t, z)|=0$.

ProOF. The implication (b) $\Rightarrow(\mathrm{a})$ is trivial. To attack the other one, assume (b) does not hold, that is,

$$
\liminf _{t \rightarrow 1^{-}} \inf _{z \in \overline{\mathbf{D}}}(1-t) \log |f(t, z)|=-\alpha
$$

for some $\alpha \in(0, \infty]$. Then there is a sequence $\left\{t_{n}\right\}_{0}^{\infty}, t_{n} \nearrow 1$, such that

$$
\lim _{n \rightarrow \infty} \inf _{z \in \overline{\mathbf{D}}}\left(1-t_{n}\right) \log \left|f\left(t_{n}, z\right)\right|=-\alpha .
$$

By the maximum modulus principle, there is a $z_{n} \in \mathbf{T}$ such that

$$
\left|f\left(t_{n}, z_{n}\right)\right|=\inf _{z \in \overline{\mathbf{D}}}\left|f\left(t_{n}, z\right)\right| .
$$

Replacing $\left\{z_{n}\right\}$ by a subsequence, we may assume that $z_{n}$ converges to some $w_{0} \in \mathbf{T}$ as $n \rightarrow \infty$. If we apply Lemma 1.1 to the functions $f_{n}(z)=f\left(z, z_{n}\right)$ and $f(z)=$ $f\left(z, w_{0}\right)$, we conclude that

$$
\lim _{t \rightarrow 1^{-}}(1-t) \log \left|f\left(t, w_{0}\right)\right| \leq-\alpha,
$$

so (a) cannot hold either. 
For $\varsigma \in \mathbf{D}$ and $f \in H^{\infty}(\mathbf{D})$, introduce the notation

$$
T_{\varsigma} f(z)=(f(z)-f(\varsigma)) /(z-\varsigma), \quad z \in \mathbf{D} \backslash\{\varsigma\} .
$$

LEMma 1.3. Assume $f \in H^{\infty}(\mathbf{D}),\|f\| \leq 1$, and that $f$ has no zeros on $\mathbf{D}$. Then

$$
\log \left\|T_{\varsigma} f / f(\varsigma)\right\| \leq 2(1+\log 1 /|f(0)|)(1-|\zeta|)^{-1}, \quad \zeta \in \mathbf{D} .
$$

Proof. Since $\|f\| \leq 1$,

$$
\log \left\|T_{\varsigma} f\right\| \leq \log \frac{2}{1-|\varsigma|} \leq \frac{2}{1-|\varsigma|}, \quad \varsigma \in \mathbf{D} .
$$

By the Herglotz representation, $\log 1 /|f(z)|=P_{z}[\mu]$ for some nonnegative Borel measure $\mu$ on $\mathbf{T}$. In particular, $\mu(\mathbf{T})=\log 1 /|f(0)|$. Now

$$
P_{\varsigma}\left(e^{i \theta}\right) \leq 2 /(1-|\zeta|), \quad \varsigma \in \mathbf{D},
$$

and hence

$$
\begin{aligned}
\log 1 /|f(\varsigma)| & =\int_{-\pi}^{\pi} P_{\zeta}\left(e^{i \theta}\right) d \mu\left(e^{i \theta}\right) \leq 2 \mu(\mathbf{T}) /(1-|\varsigma|) \\
& =2 \log 1 /|f(0)| \cdot(1-|\varsigma|)^{-1} .
\end{aligned}
$$

If we combine this estimate with (1.1), the assertion follows.

2. The bidisc algebra. The bidisc algebra $A\left(\mathbf{D}^{2}\right)$ consists of all continuous functions on $\overline{\mathbf{D}}^{2}$ that are analytic on $\mathbf{D}^{2}$. Let $f \in A\left(\mathbf{D}^{2}\right)$ have $Z(f) \subset\{1\} \times \overline{\mathbf{D}}$. Our question for the moment is: For which $f$ does it happen that the closure $I(f)$ of the principal ideal generated by $f$ contains $I(\{1\} \times \overline{\mathbf{D}})=\left\{g \in A\left(\mathbf{D}^{2}\right): g=0\right.$ on $\{1\} \times \overline{\mathbf{D}}\}$ ? For fixed $z \in \overline{\mathbf{D}}, f(\cdot, z)$ is a function in the disc algebra $A(\mathbf{D})$ which either vanishes at the point 1 only or not at all. Unless $f(\cdot, z)$ is an outer function for all $z \in \overline{\mathbf{D}}$, there is a $z_{0} \in \overline{\mathbf{D}}$ such that $f\left(\cdot, z_{0}\right) \in k_{\alpha} H^{\infty}(\mathbf{D})$ for some $\alpha>0$, where

$$
k_{\alpha}(z)=\exp (\alpha(z+1) /(z-1)), \quad z \in \mathbf{D} .
$$

Since $k_{\alpha}$ is inner, $k_{\alpha} H^{\infty}(\mathbf{D})$ is a closed ideal in $H^{\infty}(\mathbf{D})$, and hence every function $g \in I(f)$ must have $g\left(\cdot, z_{0}\right) \in k_{\alpha} H^{\infty}(\mathbf{D})$, too, which clearly prevents $I(f)$ from containing $I(\{1\} \times \overline{\mathbf{D}})$. Hence a necessary condition is that the functions $f(\cdot, z)$ should be outer for all $z \in \overline{\mathbf{D}}$. The surprising ingredient of the following theorem is that this condition is also sufficient.

THEOREM 2.1. Let $f \in A\left(\mathbf{D}^{2}\right)$ have $Z(f) \subset\{1\} \times \overline{\mathbf{D}}$. Then $I(f) \supset I(\{1\} \times \overline{\mathbf{D}})$ if and only if the functions $f(\cdot, z)$ are outer for all $z \in \overline{\mathbf{D}}$.

ProOF. The necessity is clear by the previous discussion. To prove the sufficiency, assume $f(\cdot, z)$ is outer for all $z \in \overline{\mathbf{D}}$. We wish to show that $I(f) \supset$ $I(\{1\} \times \overline{\mathbf{D}})$. As has been mentioned earlier, our assumption that $f(\cdot, z)$ is outer is equivalent to

$$
\lim _{t \rightarrow 1^{-}}(1-t) \log |f(t, z)|=0 .
$$

By Proposition 1.2,

$$
\lim _{t \rightarrow 1^{-}} \inf _{z \in \overline{\mathbf{D}}}(1-t) \log |f(t, z)|=0
$$


that is,

$$
\lim _{t \rightarrow 1^{-}}(1-t) \log \left\|f(t, \cdot)^{-1}\right\|_{\infty}=0 .
$$

Let $z_{1}$ denote the first coordinate function $z_{1}(w)=w_{1}, w=\left(w_{1}, w_{2}\right) \in \mathbf{C}^{2}$. Inspired by [DOM], we define the Carleman transform for $\phi \in A\left(\mathbf{D}^{2}\right)^{*}, \phi \perp I(f)$, to be the function

$$
\Phi(\varsigma)=\left\langle\left(\varsigma-z_{1}+I(f)\right)^{-1}, \phi\right\rangle, \quad \varsigma \in \mathbf{C} \backslash\{1\},
$$

which is holomorphic on its domain of definition, because $\left(\varsigma-z_{1}+I(f)\right)^{-1}$ has a convergent power series expansion in a neighborhood of each point $\varsigma \in \mathbf{C} \backslash\{1\}$. For $\varsigma \in \mathbf{C} \backslash \overline{\mathbf{D}}, \Phi(\varsigma)=\left\langle\left(\varsigma-z_{1}\right)^{-1}, \phi\right\rangle$, so

$$
|\Phi(\zeta)| \leq\|\phi\| /(|\zeta|-1) \text {. }
$$

We want to estimate $\Phi$ inside $\mathbf{D}$, too. For fixed $\varsigma \in \overline{\mathbf{D}}$, we may regard $f(\varsigma, \cdot)$ as a function in $A\left(\mathbf{D}^{2}\right)$ by identifying it with the function $\left(z_{1}, z_{2}\right) \mapsto f\left(\varsigma, z_{2}\right),\left(z_{1}, z_{2}\right) \in$ $\overline{\mathbf{D}}^{2}$. Introduce the notation

$$
T_{\zeta} f\left(z_{1}, z_{2}\right)=\left(f\left(z_{1}, z_{2}\right)-f\left(\varsigma, z_{2}\right)\right) /\left(z_{1}-\varsigma\right), \quad\left(z_{1}, z_{2}\right) \in(\overline{\mathbf{D}} \backslash\{\varsigma\}) \times \overline{\mathbf{D}},
$$

for $\varsigma \in \mathbf{D}$; this is then again a function in $A\left(\mathbf{D}^{2}\right)$. It is easily checked that $T_{\zeta} f / f(\zeta, \cdot)$ is an element of the coset $\left(\varsigma-z_{1}+I(f)\right)^{-1}$ for $\varsigma \in \mathbf{D}$, so that

$$
\left\|\left(\varsigma-z_{1}+I(f)\right)^{-1}\right\| \leq\left\|T_{\varsigma} f / f(\varsigma, \cdot)\right\| .
$$

Now, by Lemma 1.3,

$$
\begin{aligned}
\left\|T_{\varsigma} f / f(\varsigma, \cdot)\right\| & =\sup _{z_{1}, z_{2} \in \mathbf{D}}\left|T_{\varsigma} f\left(z_{1}, z_{2}\right) / f\left(\varsigma, z_{2}\right)\right|=\sup _{z_{2} \in \mathbf{D}}\left\|T_{\varsigma} f\left(\cdot, z_{2}\right) / f\left(\varsigma, z_{2}\right)\right\| \\
& \leq \sup _{z_{2} \in \mathbf{D}} \exp \left\{2\left(1+\log 1 /\left|f\left(0, z_{2}\right)\right|\right) \cdot(1-|\zeta|)^{-1}\right\} \\
& \leq \exp \left\{M(1-|\zeta|)^{-1}\right\}, \quad \varsigma \in \mathbf{D},
\end{aligned}
$$

for some suitably chosen constant $M$. So $\Phi$ satisfies the estimates

$$
\begin{aligned}
& |\Phi(\varsigma)| \leq\|\phi\| /(|\zeta|-1), \quad \varsigma \in \mathbf{C} \backslash \overline{\mathbf{D}}, \\
& |\Phi(\varsigma)| \leq\|\phi\| \cdot \exp \left\{M(1-|\zeta|)^{-1}\right\}, \quad \zeta \in \mathbf{D} .
\end{aligned}
$$

We are now in a situation where we can apply Yngve Domar's result on the existence of a largest subharmonic minorant (see [TAW, Lemma 5.8] or [BEG, Theorem $3.1]$ ), to conclude that there is a constant $A$ so that

$$
|\Phi(\varsigma)| \leq \exp (A /|1-\varsigma|), \quad \varsigma \in \mathbf{C} \backslash\{1\} .
$$

By (2.1),

$$
\left\|f(t, \cdot)^{-1}\right\|=O(\exp (\varepsilon /(1-t))), \quad \mathbf{R} \ni t \rightarrow 1^{-},
$$

for every $\varepsilon>0$, and since $|\Phi(\zeta)| \leq\|\phi\| \cdot\left\|T_{\zeta} f\right\| \cdot\left\|f(\zeta, \cdot)^{-1}\right\|$ and $\left\|T_{\zeta} f\right\| \leq 2\|f\|$. $(1-|\zeta|)^{-1}$ for $\zeta \in \mathbf{D}$, it follows that

$$
|\Phi(t)|=O(\exp (\varepsilon /(1-t))), \quad \mathbf{R} \ni t \rightarrow 1^{-},
$$

for all $\varepsilon>0$. Let $\gamma: \mathbf{C} \cup\{\infty\} \rightarrow \mathbf{C} \cup\{\infty\}$ be the Möbius mapping $\gamma(w)=$ $(w+i) /(w-i)$, which takes the open lower half-plane $\Pi_{-}$onto $\mathbf{D}$, and $\infty$ onto 1 . 
Since $\Phi$ is bounded at $\infty$, the function $\Psi=\Phi \circ \gamma$ is entire. Our estimates obtained for $\Phi$ translate into

$$
|\Psi(w)|=O(\exp (A|w| / 2)), \quad|w| \rightarrow \infty
$$

and

$$
|\Psi(-i v)|=O(\exp (\varepsilon v)), \quad v \rightarrow+\infty
$$

for every $\varepsilon>0$. The estimate $|\Phi(\zeta)| \leq\|\phi\| /(|\zeta|-1)$ for $|\zeta|>1$ becomes

$$
|\Psi(w)| \leq C \cdot\left(1+|w|^{2}\right) / \operatorname{Im} w, \quad \operatorname{Im} w>0,
$$

where $C$ is a constant. Introduce the function

$$
\Lambda(w)=\Psi(w) /(w-2 i)^{2}, \quad w \in \mathbf{C} \backslash\{2 i\} .
$$

By (2.4), $\Lambda(u+i)$ is bounded for $u \in \mathbf{R}$, and by $\left(2.2^{\prime}\right)-\left(2.3^{\prime}\right)$ we may apply the Phragmén-Lindelöf principle to deduce that $\Lambda$ is bounded throughout the region $\Pi_{-}+i=\{w \in \mathbf{C}: \operatorname{Im} w<1\}$. Hence we have an estimate $|\Psi(w)| \leq C\left(1+|w|^{2}\right)$ on $\Pi_{-}+i$, and since $\Psi$ has a similar bound outside $\Pi_{-}+i$ by $(2.4)$, it must be a polynomial of degree $\leq 2$. But $\Psi(i v)=O(v)$ as $v \rightarrow+\infty$, again by (2.4), which cannot be unless the degree of $\Psi$ is actually $\leq 1$. Then

$$
\Psi(w)=\beta(w-i), \quad w \in \mathbf{C},
$$

for some $\beta \in \mathbf{C}$, if we recall that $\Psi(i)=0$, so that

$$
\Phi(\varsigma)=\lambda /(\varsigma-1), \quad \varsigma \in \mathbf{C} \backslash\{1\}
$$

where $\lambda=2 i \beta$. This has the interpretation that $\phi$ acts as $\lambda$ times the "point evaluation at $z_{1}=1$ " on all the functions $\left(\varsigma-z_{1}\right)^{-1}$ for $\varsigma \in \mathbf{C} \backslash \overline{\mathbf{D}}$, and by continuity on the closure of the linear span of such functions, which consists of those functions that only depend on the first variable $z_{1}$. In particular, $z_{1}-1$ is such a function, so that $\left\langle z_{1}-1, \phi\right\rangle=0$. Since $\phi \perp I(f)$ was arbitrary, it follows that $z_{1}-1 \in I(f)$. The assertion follows, because the closure of the principal ideal generated by $z_{1}-1$ equals $I(\{1\} \times \overline{\mathbf{D}})$.

COROllary 2.2. Let $f \in A\left(\mathbf{D}^{2}\right)$ have $Z(f) \subset\{1\} \times \overline{\mathbf{D}}$. Then $I(f)=I(Z(f))$ if and only if the functions $f(\cdot, z)$ are all outer for $z \in \overline{\mathbf{D}}$, and $f(1, \cdot)$ either vanishes identically or is an outer function.

ProOF. The relation $I(f)=I(Z(f))$ cannot hold unless $I(f) \supset I(\{1\} \times \overline{\mathbf{D}})$, so by Theorem 2.1 it is necessary that $f(\cdot, z)$ is an outer function for all $z \in \overline{\mathbf{D}}$. It was mentioned in the introduction that our assumption $Z(f) \subset\{1\} \times \overline{\mathbf{D}}$ forces $f(1, \cdot)$ to either vanish identically or have no zeros on $\mathbf{D}$. If, in the case $f(1, \cdot) \not \equiv 0, f(1, \cdot)$ has a noninvertible inner factor, so will every $g(1, \cdot)$ with $g \in I(f)$, which clearly prevents $I(f)$ from being equal to $I(Z(f))$.

Let us now turn to the sufficiency. Denote by $R$ the restriction mapping $g \mapsto$ $g(1, \cdot), g \in A\left(\mathbf{D}^{2}\right)$. By Theorem 2.1,I(f) $I(\{1\} \times \overline{\mathbf{D}})$, so that

$$
I(f)=\left\{g \in A\left(\mathbf{D}^{2}\right): g(1, \cdot) \in R(I(f))\right\} .
$$

If $Z(f)=\{1\} \times \overline{\mathbf{D}}$, we are done. If $f(1, \cdot)$ is an outer function, the closed $A(\mathbf{D})$ ideal $R(I(f))$ is an intersection of maximal ideals by the Beurling-Rudin theorem [RUD1, HOF], and hence $I(f)=I(Z(f))$. 
COROLlaRY 2.3. Let $f \in A\left(\mathbf{D}^{2}\right)$ have $Z(f)=\{(1,1)\}$. Then $I(f)=$ $I(\{(1,1)\})$ if and only if $f(1, \cdot)$ and $f(\cdot, 1)$ are both outer functions.

PROOF. This is a special case of Corollary 2.2 .

REMARKS 2.4. (a) The technique of this section can most likely be modified to incorporate cases when $Z(f) \subset E \times \overline{\mathbf{D}}$ for closed sets $E \subset \mathbf{T}$ of linear measure 0 .

(b) If $f \in A\left(\mathbf{D}^{2}\right), Z(f) \subset\{1\} \times \overline{\mathbf{D}}$, but

$$
\inf _{z \in \overline{\mathbf{D}}} \lim _{t \rightarrow 1^{-}}(1-t) \log |f(t, z)|=-2 \alpha<0,
$$

one can show (not without effort) that $I(f)$ contains the function

$$
\left(z_{1}, z_{2}\right) \mapsto\left(1-z_{1}\right) \exp \left(\alpha \frac{z_{1}+1}{z_{1}-1}\right), \quad\left(z_{1}, z_{2}\right) \in \mathbf{D}^{2} .
$$

(c) Given that $Z(f) \subset\{1\} \times \overline{\mathbf{D}}$, the condition on the function $f$ in Theorem 2.1 is considerably stronger than requiring that $f$ is outer in the sense that

$$
\log |f(0,0)|=(2 \pi)^{-2} \int_{\mathbf{T}^{2}} \log \left|f\left(e^{i \theta_{1}}, e^{i \theta_{2}}\right)\right| d \theta_{1} d \theta_{2} .
$$

This latter condition is equivalent to $f(\cdot, 0)$ being outer.

EXAMPLE 2.5. (a) Let $q$ be the $A(\mathbf{D})$ function

$$
q(z)=\frac{\log (2 /(1-z))}{1+\log (2 /(1-z))}, \quad z \in \mathbf{D}
$$

which peaks at 1 , and put

$$
p\left(z_{1}, z_{2}\right)=\left(z_{1}+q\left(z_{2}\right)\right) / 2, \quad\left(z_{1}, z_{2}\right) \in \mathbf{D}^{2},
$$

which peaks at $(1,1)$. Consider the bidisc algebra function

$$
f\left(z_{1}, z_{2}\right)=\left(2-z_{1}-z_{2}\right) \exp \left\{\frac{1}{2}\left(p\left(z_{1}, z_{2}\right)+1\right) /\left(p\left(z_{1}, z_{2}\right)-1\right)\right\}, \quad\left(z_{1}, z_{2}\right) \in \mathbf{D}^{2},
$$

which vanishes at the point $(1,1)$ only. It is an element of the closed ideal

$$
J=\left\{g \in A\left(\mathbf{D}^{2}\right): g(\cdot, 1) \in k H^{\infty}(\mathbf{D})\right\},
$$

where $k$ is the singular inner function

$$
k(z)=\exp ((z+1) /(z-1)), \quad z \in \mathbf{D},
$$

because $p\left(z_{1}, 1\right)=\left(1+z_{1}\right) / 2$. On the other hand, $f(1, \cdot)$ is an outer function, and since $f(z, \cdot)$ is trivially outer for $z \in \overline{\mathbf{D}} \backslash\{1\}$, Theorem 2.1 tells us that $I(f) \supset$ $I(\overline{\mathbf{D}} \times\{1\})$. Hence $I(f)=J$ by the Beurling-Rudin theorem [RUD1, HOF] applied to the algebra $A\left(\mathbf{D}^{2}\right) / I(\overline{\mathbf{D}} \times\{1\}) \cong A(\mathbf{D})$. In particular, $J$ is singly generated.

(b) Let $L: \mathbf{D} \rightarrow \mathbf{D}^{2}$ be the analytic disc $L(z)=(z, z)$, and let $k$ be as in (a). Then the closed ideal $\left\{f \in A\left(\mathbf{D}^{2}\right): f \circ L \in k H^{\infty}(\mathbf{D})\right\}$ cannot be singly generated, in view of Corollary 2.3 . 
3. The algebra $H^{\infty}\left(\mathbf{D}^{2}\right)$. We denote by $H^{\infty}\left(\mathbf{D}^{2}\right)$ the Banach algebra of bounded analytic functions on $\mathrm{D}^{2}$, supplied with the uniform norm. The maximal ideal space $\mathcal{M}\left(H^{\infty}\left(\mathbf{D}^{2}\right)\right)$ of $H^{\infty}\left(\mathbf{D}^{2}\right)$ is pretty messy; it is not even known whether $\mathbf{D}^{2}$, regarded as a subset of $\mathcal{M}\left(H^{\infty}\left(\mathbf{D}^{2}\right)\right)$ in the obvious way, is dense in $\mathcal{M}\left(H^{\infty}\left(\mathbf{D}^{2}\right)\right)$. It is known, however, that for $\alpha \in \partial \mathbf{D}^{2} \backslash \mathbf{T}^{2}$, the fiber

$$
\mathcal{M}_{\alpha}\left(H^{\infty}\left(\mathbf{D}^{2}\right)\right)=\left\{m \in \mathcal{M}\left(H^{\infty}\left(\mathbf{D}^{2}\right)\right):\left(m\left(z_{1}\right), m\left(z_{2}\right)\right)=\alpha\right\}
$$

lies in the closure of $\mathbf{D}^{2}$, by the corona theorem for $H^{\infty}(\mathbf{D})$ (see [ROS]). For $\alpha \in \mathbf{D}$, let $L_{\alpha}: \mathbf{D} \rightarrow \mathbf{D}^{2}$ be the analytic disc mapping $L_{\alpha}(z)=(z, \alpha), z \in \mathbf{D}$. It can be shown (this is implicit in [JAN, pp. $31-32 ; \mathbf{R O S}]$ ) that one can find analytic disc mappings $L_{m}: \mathbf{D} \rightarrow \mathcal{M}\left(H^{\infty}\left(\mathbf{D}^{2}\right)\right)$, varying continuously with $m \in \mathcal{M}\left(H^{\infty}(\mathbf{D})\right)$ such that $L_{m}=L_{\alpha}$ if $m=\alpha \in \mathbf{D}$. Moreover, as $\mathbf{D} \ni \alpha \rightarrow m, f \circ L_{\alpha} \rightarrow \hat{f} \circ L_{m}$ uniformly on compact subsets of $\mathbf{D}\left(\hat{f}\right.$ is the Gelfand transform of $\left.f \in H^{\infty}\left(\mathbf{D}^{2}\right)\right)$, and $\bigcup\left\{L_{m}(\mathbf{D}): m \in \mathcal{M}\left(H^{\infty}(\mathbf{D})\right)\right\}=\left\{m \in \mathcal{M}\left(H^{\infty}\left(\mathbf{D}^{2}\right)\right):\left|m\left(z_{1}\right)\right|<1\right\}$. For a function $f \in H^{\infty}\left(\mathbf{D}^{2}\right)$, let $\mathrm{Cl}(f, \alpha)$ denote the cluster set of $f$ at the point $\alpha \in \overline{\mathbf{D}}^{2}$, that is, the set of all numbers $\xi$ such that for some sequence $\mathbf{D}^{2} \ni z_{n} \rightarrow \alpha, f\left(z_{n}\right) \rightarrow$ $\xi$. Also, put $Z(\hat{f})=\left\{m \in \mathcal{M}\left(H^{\infty}\left(\mathbf{D}^{2}\right)\right): m(f)=0\right\}$, and let $I(f)$ denote the closure of the principal ideal generated by $f$. By the Cluster Set Theorem (see [GAM, p. 7]), $\mathrm{Cl}(f, \alpha)=\hat{f}\left(\mathcal{M}_{\alpha}\left(H^{\infty}\left(\mathbf{D}^{2}\right)\right)\right)$. Denote by $\mathcal{M}_{\{1\} \times \overline{\mathbf{D}}}\left(H^{\infty}\left(\mathbf{D}^{2}\right)\right)$ the union of all fibers $\mathcal{M}_{\alpha}\left(H^{\infty}\left(\mathbf{D}^{2}\right)\right)$ with $\alpha \in\{1\} \times \overline{\mathbf{D}}$, and by $I(\{1\} \times \overline{\mathbf{D}})$ the closed ideal of all functions $g \in H^{\infty}\left(\mathbf{D}^{2}\right)$ such that $g(z) \rightarrow 0$ as $z \rightarrow\{1\} \times \overline{\mathbf{D}}$. We then have the following result.

THEOREM 3.1. Let $f \in H^{\infty}\left(\mathbf{D}^{2}\right)$ be such that

$$
0 \notin \mathrm{Cl}(f, \alpha) \quad \text { for } \alpha \in \overline{\mathbf{D}}^{2} \backslash(\{1\} \times \overline{\mathbf{D}}),
$$

or in other words, assume $Z(\hat{f}) \subset \mathcal{M}_{\{1\} \times \overline{\mathbf{D}}}\left(H^{\infty}\left(\mathbf{D}^{2}\right)\right)$. Then $I(f) \supset I(\{1\} \times \overline{\mathbf{D}})$ if and only if

$$
\lim _{t \rightarrow 1^{-}} \inf _{z \in \mathbf{D}}(1-t) \log |f(t, z)|=0 .
$$

REMARK 3.2. Condition (3.1) is equivalent to $\hat{f} \circ L_{m}$ being outer for all $m \in$ $\mathcal{M}\left(H^{\infty}(\mathbf{D})\right)$, as will become apparent from the proof of the theorem.

PROOF OF THE THEOREM. The equivalence of the conditions on the cluster sets of $f$ and $Z(\hat{f})$ follows from the Cluster Set Theorem (see [GAM, p. 7]). Let us first prove the sufficiency, that is, that (3.1) implies $I(f) \supset I(\{1\} \times \overline{\mathbf{D}})$. Condition (3.1) can be restated as

$$
\lim _{t \rightarrow 1^{-}}(1-t) \log \left\|f(t, \cdot)^{-1}\right\|_{\infty}=0 .
$$

Just as in the proof of Theorem 2.1, we pick a continuous linear functional $\phi \in$ $H^{\infty}\left(\mathbf{D}^{2}\right)^{*}, \phi \perp I(f)$, and consider its Carleman transform

$$
\Phi(\zeta)=\left\langle\left(\varsigma-z_{1}+I(f)\right)^{-1}, \phi\right\rangle,
$$

which is well defined for $\varsigma \in \mathbf{C} \backslash\{1\}$ because of our condition

$$
Z(\hat{f}) \subset \mathcal{M}_{\{1\} \times \overline{\mathbf{D}}}\left(H^{\infty}\left(\mathbf{D}^{2}\right)\right),
$$


and it is holomorphic there, since $(\varsigma-z+I(f))^{-1}$ has a convergent power series expansion near each point $\varsigma \in \mathbf{C} \backslash\{1\}$. Since the norms on $A\left(\mathbf{D}^{2}\right)$ and $H^{\infty}\left(\mathbf{D}^{2}\right)$ are the same, we can get the same estimates of $\Phi$ as we did back in the proof of Theorem 2.1, so we can use (3.2) to show that

$$
\Phi(\varsigma)=\lambda /(\varsigma-1), \quad \varsigma \in \mathbf{C} \backslash\{1\},
$$

for some $\lambda \in \mathbf{C}$, which in its turn forces $z_{1}-1$ to be in $I(f)$. But the closure of the principal ideal generated by $z_{1}-1$ equals $I(\{1\} \times \overline{\mathbf{D}})$, and hence $I(\{1\} \times \overline{\mathbf{D}}) \subset I(f)$.

Let us turn to the necessity part. In order for $I(f)$ to contain $I(\{1\} \times \overline{\mathbf{D}})$, it is necessary that the functions $\hat{f} \circ L_{m}$ are all outer for $m \in \mathcal{M}\left(H^{\infty}(\mathbf{D})\right)$, simply because otherwise $I(f)$ could not possibly contain the function $z_{1}-1$. We intend to show that this condition implies (3.1); what is needed is a generalization to $H^{\infty}\left(\mathbf{D}^{2}\right)$ of Proposition 1.2. Assume that (3.1) does not hold, that is,

$$
\lim _{t \rightarrow 1^{-}} \inf _{z \in \mathbf{D}}(1-t) \log |f(t, z)|=-\delta
$$

for some $\delta, 0<\delta \leq+\infty$. Then there are two sequences $\left\{t_{n}\right\}_{0}^{\infty} \subset[0,1)$ and $\left\{z_{n}\right\}_{0}^{\infty} \subset \mathbf{D}$, with $t_{n} \nearrow 1$ and $z_{n} \rightarrow w \in \mathbf{T}$ as $n \rightarrow \infty$, such that

$$
\lim _{n \rightarrow \infty}\left(1-t_{n}\right) \log \left|f\left(t_{n}, z_{n}\right)\right|=-\delta \text {. }
$$

Put $f_{n}(z)=f\left(z, z_{n}\right)$. By Lemma 1.1,

$$
\lim _{t \rightarrow 1^{-}}(1-t) \log |g(t)| \leq-\delta
$$

for every normal limit $g$ of $\left\{f_{n}\right\}_{0}^{\infty}$, because our assumption on the cluster sets of $f$ excludes the possibility that $g$ could vanish anywhere on $\mathbf{D}$. If $m \in \mathcal{M}\left(H^{\infty}(\mathbf{D})\right)$ is a cluster point for the sequence $\left\{z_{n}\right\}_{0}^{\infty}, \hat{f} \circ L_{m}$ is a normal limit of the functions $f \circ L_{z_{n}}=f_{n}$. so by $(3.3), \hat{f} \circ L_{m}$ cannot possibly be an outer function. Hence $I(f)$ in its turn cannot contain $I(\{1\} \times \overline{\mathbf{D}})$, and the proof of the theorem is complete.

4. A weighted Hilbert space. Let $l_{1,1}^{2}\left(\mathbf{Z}^{2}\right)$ be the Hilbert space of all complex-valued sequences $\left\{a_{k n}\right\}_{k, n \in \mathbf{Z}}$ that satisfy

$$
\sum_{k, n \in \mathbf{Z}}\left(k^{2}+1\right)\left(n^{2}+1\right)\left|a_{k n}\right|^{2}<\infty
$$

and let this expression be the square of the norm on $l_{1,1}^{2}\left(\mathbf{Z}^{2}\right)$. It is easy to see that $l_{1,1}^{2}\left(\mathbf{Z}^{2}\right) \subset l^{1}\left(\mathbf{Z}^{2}\right)$, so that we can associate to each $\left\{a_{k n}\right\}_{k, n \in \mathbf{Z}}$ in $l_{1,1}^{2}\left(\mathbf{Z}^{2}\right)$ its Fourier series

$$
f(z)=\sum_{k, n} a_{k n} z_{1}^{k} z_{2}^{n}, \quad z=\left(z_{1}, z_{2}\right) \in \mathbf{T}^{2},
$$

which is a continuous function on $\mathbf{T}^{2}$. When equipped with sequence convolution, $l_{1,1}^{2}\left(\mathbf{Z}^{2}\right)$ is a commutative Banach algebra (see [TCH, pp. 152-154], for a proof in the continuous case; the same proof works in our situation also). Its maximal ideal space is $\mathbf{T}^{2}$, and the Gelfand transform of a sequence is its Fourier series. The image of $l_{1,1}^{2}\left(\mathbf{Z}^{2}\right)$ under the Gelfand transformation is the space $L_{1,1}^{2}\left(\mathbf{T}^{2}\right)$ of functions $f \in L^{2}\left(\mathbf{T}^{2}\right)$ for which $\partial f / \partial z_{1}, \partial f / \partial z_{2}, \partial^{2} f / \partial z_{1} \partial z_{2} \in L^{2}\left(\mathbf{T}^{2}\right)$ (if $z_{1}=e^{i \theta_{1}}, \partial / \partial z_{1}$ is the operator $\left.-i e^{-i \theta_{1}} \partial / \partial \theta_{1}\right)$. Consider the closed subalgebra $l_{1,1}^{2}\left(\mathbf{N}^{2}\right)$ of $L_{1,1}^{2}\left(\mathbf{Z}^{2}\right)$ consisting of those sequences that are supported on $\mathbf{N}^{2}$; 
here, and always, $\mathbf{N}=\{0,1,2, \ldots\}$. For sequences in $l_{1,1}^{2}\left(\mathbf{N}^{2}\right)$, the Fourier series is a Taylor series, and the maximal ideal space of $l_{1,1}^{2}\left(\mathbf{N}^{2}\right)$ is $\overline{\mathbf{D}}^{2}$. The Gelfand transformation takes $l_{1,1}^{2}\left(\mathbf{N}^{2}\right)$ onto $H_{1,1}^{2}\left(\mathbf{D}^{2}\right)$, the space of all functions $f \in H^{2}\left(\mathbf{D}^{2}\right)$ with $\partial f / \partial z_{1}, \partial f / \partial z_{2}, \partial^{2} f / \partial z_{1} \partial z_{2} \in H^{2}\left(\mathbf{D}^{2}\right)$; observe that $H_{1,1}^{2}\left(\mathbf{D}^{2}\right) \subset A\left(\mathbf{D}^{2}\right)$. We are interested in those closed subspaces of $l_{1,1}^{2}\left(\mathbf{N}^{2}\right)$ that are invariant under right and upper translations, or which is the same, the closed ideals of $H_{1,1}^{2}\left(\mathbf{D}^{2}\right)$. For a function $f \in H_{1,1}^{2}\left(\mathbf{D}^{2}\right)$ and a set $E \subset \overline{\mathbf{D}}^{2}$, let $I(f)$ denote the closure of the principal ideal generated by $f$, and put

$$
I(E)=\left\{g \in H_{1,1}^{2}\left(\mathbf{D}^{2}\right): g=0 \text { on } E\right\} .
$$

THEOREM 4.1. Let $f \in H_{1,1}^{2}\left(\mathbf{D}^{2}\right)$ have $Z(f) \subset\{1\} \times \overline{\mathbf{D}}$. Then $I(f) \supset$ $I(\{1\} \times \overline{\mathbf{D}})$ if and only if $f(\cdot, z)$ is an outer function for all $z \in \overline{\mathbf{D}}$.

PROOF. The necessity of the condition is obvious in view of the discussion preceding Theorem 2.1 .

Our proof will mimic that of Theorem 2.1. All we need to do is to check some crucial points. For a functional $\phi \in H_{1,1}^{2}\left(\mathbf{D}^{2}\right)^{*}$ that annihilates $I(f)$, put

$$
\Phi(\varsigma)=\left\langle\left(\varsigma-z_{1}+I(f)\right)^{-1}, \phi\right\rangle, \quad \varsigma \in \mathbf{C} \backslash\{1\},
$$

which is a holomorphic function on $\mathbf{C} \backslash\{1\}$. Outside the unit disc we have the estimate

$$
|\Phi(\varsigma)| \leq\|\phi\| \cdot\left\|\left(\varsigma-z_{1}\right)^{-1}\right\| \leq 2\|\phi\| \cdot(|\varsigma|-1)^{-3 / 2}, \quad \varsigma \in \mathbf{C} \backslash \overline{\mathbf{D}} .
$$

If $T_{\varsigma} f$ is the function defined in the proof of Theorem 2.1 for $\varsigma \in \mathbf{D}$, then $T_{\varsigma} f \in$ $H_{1,1}^{2}\left(\mathbf{D}^{2}\right)$ since it is in both $A\left(\mathbf{D}^{2}\right)$ and $L_{1,1}^{2}\left(\mathbf{T}^{2}\right)$. Hence $T_{\varsigma} f / f(\varsigma, \cdot)$ is an element of the coset $\left(\varsigma-z_{1}+I(f)\right)^{-1}$, so that

$$
|\Phi(\varsigma)| \leq\|\phi\| \cdot\left\|T_{\varsigma} f / f(\varsigma, \cdot)\right\|, \quad \varsigma \in \mathbf{D} .
$$

It is not hard to see that

$$
\left\|T_{\varsigma} f\right\| \leq 4\|f\|(1-|\varsigma|)^{-3 / 2}, \quad \varsigma \in \mathbf{D}
$$

just evaluate the $L_{1,1}^{2}\left(\mathbf{T}^{2}\right)$-norm of $T_{\varsigma} f$. The final estimate we need is the following:

$$
\left\|f(\zeta, \cdot)^{-1}\right\| \leq C\left(\left\|f(\zeta, \cdot)^{-1}\right\|_{\infty}+\left\|f(\zeta, \cdot)^{-1}\right\|_{\infty}^{2}\right), \quad \zeta \in \mathbf{D} .
$$

However, this follows from the fact that

$$
\partial / \partial z_{2}\left(1 / f\left(\varsigma, z_{2}\right)\right)=-\partial f / \partial z_{2}\left(\varsigma, z_{2}\right) /\left(f\left(\varsigma, z_{2}\right)\right)^{2},
$$

so that

$$
\begin{aligned}
\left\|f(\zeta, \cdot)^{-1}\right\| & \leq\left\|f(\zeta, \cdot)^{-1}\right\|_{H^{2}}+\left\|\partial f / \partial z_{2}(\zeta, \cdot)\right\|_{H^{2}} \cdot\left\|f(\zeta, \cdot)^{-1}\right\|_{\infty}^{2} \\
& \leq\left\|f(\zeta, \cdot)^{-1}\right\|_{\infty}+2\|f\| \cdot\left\|f(\zeta, \cdot)^{-1}\right\|_{\infty}^{2} .
\end{aligned}
$$

The point now is that these estimates enable us to pull through the proof of Theorem 2.1 to the point where $z_{1}-1 \in I(f)$. The last step, to show that $I\left(z_{1}-1\right)=$ $I(\{1\} \times \overline{\mathbf{D}})$, is slightly more complicated here because the norm is no longer uniform. First, observe that a polynomial that vanishes on $\{1\} \times \overline{\mathbf{D}}$ is divisible by $z_{1}-1$, and hence lies in $I\left(z_{1}-1\right)$. Now let $g \in I(\{1\} \times \overline{\mathbf{D}})$ be arbitrary. Because the polynomials are dense in $H_{1,1}^{2}\left(\mathbf{D}^{2}\right)$, there is a polynomial $P$ such that $\|g-P\|<\varepsilon$. If we put $Q\left(z_{1}, z_{2}\right)=P\left(z_{1}, z_{2}\right)-P\left(1, z_{2}\right)$, then $\|g-Q\|<3 \varepsilon$ and $Q \in I\left(z_{1}-1\right)$. Hence $g \in I\left(z_{1}-1\right)$, and the proof is finished. 
COROLlary 4.2. Let $f \in H_{1,1}^{2}\left(\mathbf{D}^{2}\right)$ have $Z(f) \subset\{1\} \times \mathbf{D}$. Then $I(f)=$ $I(Z(f))$ if and only if the functions $f(\cdot, z)$ are all outer for $z \in \bar{D}$, and $f(1, \cdot)$ either vanishes identically or is an outer function.

PROOF. Mimic the proof of Corollary 2.2, and replace the Beurling-Rudin Theorem by Boris Korenblum's [KOR1] description of the closed ideals of the algebra $H_{1}^{2}(\mathbf{D})=\left\{f \in H^{2}(\mathbf{D}): f^{\prime} \in H^{2}(\mathbf{D})\right\}$.

Corollary 4.3. Let $f \in H_{1,1}^{2}\left(\mathbf{D}^{2}\right)$ have $Z(f)=\{(1,1)\}$. Then $I(f)=$ $I(\{(1,1)\})$ if and only if $f(1, \cdot)$ and $f(\cdot, 1)$ are both outer functions.

PROOF. This is a special case of Corollary 4.2 .

5. Translation invariance in $L^{1}\left(\mathbf{R}_{+}^{2}\right)$. The space $L^{\mathbf{1}}\left(\mathbf{R}^{2}\right)$ is a Banach algebra when equipped with convolution multiplication:

$$
f * g\left(x_{1}, x_{2}\right)=\int_{\mathbf{R}^{2}} f\left(x_{1}-t_{1}, x_{2}-t_{2}\right) g\left(t_{1}, t_{2}\right) d t_{1} d t_{2}, \quad\left(x_{1}, x_{2}\right) \in \mathbf{R}^{2},
$$

for $f, g \in L^{1}\left(\mathbf{R}^{2}\right)$. For $x \in \mathbf{R}^{2}$, let $T_{x}$ be the translation operator

$$
T_{x} f(t)=f(t-x), \quad t \in \mathbf{R}^{2} .
$$

A generalized version [GRS, §40] of Wiener's Tauberian Theorem states that in order for the translates $T_{x} f, x \in \mathbf{R}^{2}$, of a given function $f \in L^{1}\left(\mathbf{R}^{2}\right)$ to span a dense subspace of $L^{1}\left(\mathbf{R}^{2}\right)$ it is necessary and sufficient that the Fourier transform

$$
\hat{f}\left(z_{1}, z_{2}\right)=\int_{\mathbf{R}^{2}} e^{-i t_{1} z_{1}-i t_{2} z_{2}} f\left(t_{1}, t_{2}\right) d t_{1} d t_{2}, \quad\left(z_{1}, z_{2}\right) \in \mathbf{R}^{2},
$$

of $f$ does not vanish anywhere on $\mathbf{R}^{2}$. The corresponding question for the closed subalgebra $L^{1}\left(\mathbf{R}_{+}^{2}\right)$ of functions vanishing (almost everywhere) on $\mathbf{R}^{2} \backslash \mathbf{R}_{+}^{2}$, where $\mathbf{R}_{+}^{2}=\mathbf{R}_{+} \times \mathbf{R}_{+}$and $\mathbf{R}_{+}=(0, \infty)$, is still unsolved. More precisely, the problem is: Give necessary and sufficient conditions on a function $f \in L^{1}\left(\mathbf{R}_{+}^{2}\right)$ so that its combined right and upper translates $T_{x} f, x \in \mathbf{R}_{+}^{2}$, span a dense subspace of $L^{1}\left(\mathbf{R}_{+}^{2}\right)$. This problem was raised a long time ago by B. Ya. Levin, and appeared recently in [LEV]. An obvious necessary condition is the nonvanishing of the Fourier transform, which for $f \in L^{1}\left(\mathbf{R}_{+}^{2}\right)$ is a holomorphic function on the region $\Pi_{-}^{2}=$ $\Pi_{-} \times \Pi_{-}$, where $\Pi_{-}$is the lower half-plane $\{z \in \mathbf{C}: \operatorname{Im} z<0\}$, which extends continuously to the boundary and tends to 0 at infinity. However, this condition is far from being sufficient. It is not even sufficient in the one-dimensional case, which was completely solved by Bertil Nyman [NYM]: the right translates $T_{x} f, x \in \mathbf{R}_{+}$, of a function $f \in L^{1}\left(\mathbf{R}_{+}\right)$span a dense subspace of $L^{1}\left(\mathbf{R}_{+}\right)$if and only if

(a) $\hat{f}(z) \neq 0$ for all $z \in \bar{\Pi}_{-}$, and

(b) there is no interval $(0, \alpha), \alpha>0$, such that $f$ vanishes almost everywhere on $(0, \alpha)$, or in other words,

$$
\lim _{t \rightarrow+\infty} t^{-1} \log |\hat{f}(-i t)|=0 .
$$

Denote by $A_{0}\left(\Pi_{-}^{2}\right)$ the Banach algebra of holomorphic functions on $\Pi_{-}^{2}$ that extend continuously to $\bar{\Pi}_{-}^{2} \cup\{\infty\}$ and have value 0 at $\infty$. The Fourier transform defines a monomorphism (injective homomorphism) $L^{1}\left(\mathbf{R}_{+}^{2}\right) \rightarrow A_{0}\left(\Pi_{-}^{2}\right)$ with dense 
range. After a Möbius transformation, we may identify $A_{0}\left(\Pi_{-}^{2}\right)$ with the closed $A\left(\mathrm{D}^{2}\right)$-ideal

$$
I_{0}=\left\{f \in A\left(\mathbf{D}^{2}\right): f=0 \text { on }(\{1\} \times \overline{\mathbf{D}}) \cup(\overline{\mathbf{D}} \times\{1\})\right\} .
$$

Since a closed translation invariant subspace of $L^{1}\left(\mathbf{R}_{+}^{2}\right)$ is the same as a closed ideal, a uniform norm version of Levin's problem would ask for a description of those functions $f \in I_{0}$ which generate an ideal that is dense in $I_{0}$. In this paper, we are only able to deal with smaller zero sets like $\{1\} \times \overline{\mathbf{D}}$. Therefore we will choose to work with a bigger algebra than $L^{1}\left(\mathbf{R}_{+}^{2}\right)$, namely $L^{1}\left(\mathbf{R}_{+}^{2}, \mathbf{R}_{+}\right)=L^{1}\left(\mathbf{R}_{+}^{2}\right) \oplus L^{1}\left(\mathbf{R}_{+}\right)$, consisting of pairs $(f, g)$ with $f \in L^{1}\left(\mathbf{R}_{+}^{2}\right)$ and $g \in L^{1}\left(\mathbf{R}_{+}\right)$. The convolution product of two elements $(f, 0)$ and $(0, g)$ is defined to be the $L^{1}\left(\mathbf{R}_{+}^{2}\right)$ function

$$
((f, 0) *(0, g))\left(x_{1}, x_{2}\right)=\int_{0}^{\infty} f\left(x_{1}-t, x_{2}\right) g(t) d t .
$$

On $L^{1}\left(\mathbf{R}_{+}^{2}\right)$ and $L^{1}\left(\mathbf{R}_{+}\right)$, regarded as subspaces of $L^{1}\left(\mathbf{R}_{+}^{2}, \mathbf{R}_{+}\right)$, the product is usual convolution. We can think of $L^{1}\left(\mathbf{R}_{+}^{2}, \mathbf{R}_{+}\right)$as the space of those finite Borel measures on $\mathbf{R}_{+} \times\left(\mathbf{R}_{+} \cup\{0\}\right)$ that are absolutely continuous with respect to area measure on $\mathbf{R}_{+}^{2}$, and absolutely continuous with respect to linear measure on $\mathbf{R}_{+} \times$ $\{0\}$. For $f=\left(f_{1}, f_{2}\right) \in L^{1}\left(\mathbf{R}_{+}^{2}, \mathbf{R}_{+}\right)$, its Fourier transform

$$
\begin{aligned}
\hat{f}(z)= & \int_{0}^{\infty} \int_{0}^{\infty} f_{1}\left(t_{1}, t_{2}\right) e^{-i t_{1} z_{1}-i t_{2} z_{2}} d t_{1} d t_{2} \\
& +\int_{0}^{\infty} f_{2}\left(t_{1}\right) e^{-i t_{1} z_{1}} d t_{1}, \quad z=\left(z_{1}, z_{2}\right) \in \bar{\Pi}_{-}^{2},
\end{aligned}
$$

extends continuously to $\left(\bar{\Pi}_{-} \cup\{\infty\}\right) \times\left(\bar{\Pi}_{-} \cup\{\infty\}\right)$ in such a way that $\hat{f}\left(\infty, z_{2}\right) \equiv 0$ and

$$
\hat{f}\left(z_{1}, \infty\right)=\int_{0}^{\infty} f_{2}\left(t_{1}\right) e^{-i t_{1} z_{1}} d t_{1}, \quad z_{1} \in \bar{\Pi}_{-} .
$$

Let

$$
\mathcal{F}_{2} f\left(t_{1}, z_{2}\right)=\int_{0}^{\infty} f_{1}\left(t_{1}, t_{2}\right) e^{-i t_{2} z_{2}} d t_{2}+f_{2}\left(t_{1}\right), \quad t_{1} \in \mathbf{R}_{+}, z_{2} \in \bar{\Pi}_{-},
$$

be the partial Fourier transform of $f$ with respect to the second coordinate, which extends continuously to $z_{2}=\infty$ :

$$
\mathcal{F}_{2} f\left(t_{1}, \infty\right)=f_{2}\left(t_{1}\right), \quad t_{1} \in \mathbf{R}_{+} .
$$

Moreover, let $I(f)$ denote the closure of the principal ideal generated by $f$.

THEOREM 5.1. Let $f=\left(f_{1}, f_{2}\right) \in L^{1}\left(\mathbf{R}_{+}^{2}, \mathbf{R}_{+}\right)$, and assume

$$
\int_{0}^{\infty}\left(1+t_{2}^{2}\right)\left(\int_{0}^{\infty}\left|f_{1}\left(t_{1}, t_{2}\right)\right| d t_{1}\right)^{2} d t_{1}<\infty
$$

Then $I(f)=L^{1}\left(\mathbf{R}_{+}^{2}, \mathbf{R}_{+}\right)$if and only if

(a) $\hat{f}(z) \neq 0$ for all $z \in \bar{\Pi}_{-} \times\left(\bar{\Pi}_{-} \cup\{\infty\}\right)$, and

(b) for all $z_{2} \in \bar{\Pi}_{-} \cup\{\infty\}$, the $L^{1}\left(\mathbf{R}_{+}\right)$function $t_{1} \mapsto \mathcal{F}_{2} f\left(t_{1}, z_{2}\right)$ does not vanish almost everywhere on any interval $(0, \alpha), \alpha>0$.

OUTLINE OF PROOF. Clearly (a) (b) are necessary. To obtain the sufficiency, let $L_{e}^{1}\left(\mathbf{R}_{+}^{2}, \mathbf{R}_{+}\right)$be the unitization of $L^{1}\left(\mathbf{R}_{+}^{2}, \mathbf{R}_{+}\right)$; the unit can be identified with the 
Dirac measure $\delta_{0}$ at the point $(0,0)$. Put $a=\left(0, a_{2}\right)$, where $a_{2}(t)=e^{-t}, t \in \mathbf{R}_{+}$. The Fourier transform of $a$ is

$$
\hat{a}\left(z_{1}, z_{2}\right)=1 /\left(1+i z_{1}\right), \quad\left(z_{1}, z_{2}\right) \in \bar{\Pi}_{-}^{2},
$$

and since $\hat{a}$ extends to be zero on $\{\infty\} \times\left(\bar{\Pi}_{-} \cup\{\infty\}\right)$, the element $\left(\zeta \delta_{0}-a+I(f)\right)$ is invertible in the quotient algebra $L_{e}^{1}\left(\mathbf{R}_{+}^{2}, \mathbf{R}_{+}\right) / I(f)$ for all $\varsigma \in \mathbf{C} \backslash\{0\}$, by (a). Let $\phi=\left(\phi_{1}, \phi_{2}\right) \in L^{\infty}\left(\mathbf{R}_{+}^{2}\right) \oplus L^{\infty}\left(\mathbf{R}_{+}\right)=\left(L^{1}\left(\mathbf{R}_{+}^{2}, \mathbf{R}_{+}\right)\right)^{*}$ annihilate $I(f)$, and extend $\phi$ to $L_{e}^{1}\left(\mathbf{R}_{+}^{2}, \mathbf{R}_{+}\right)$by defining $\left\langle\delta_{0}, \phi\right\rangle=0$. Consider the function

$$
\Phi(\varsigma)=\left\langle\left(\varsigma \delta_{0}-a+I(f)\right)^{-1}, \phi\right\rangle, \quad \varsigma \in \mathbf{C} \backslash\{0\},
$$

which is analytic where it is defined. After composing $\Phi$ with a Möbius mapping that sends the point 0 to infinity and some algebraic manipulations (see [DOM or HED, pp. 134. 137]), we obtain an entire function $\Psi$ for which

$$
\Psi(z)=\int_{0}^{\infty} e^{i t z} \phi_{2}(t) d t, \quad \operatorname{Im} z>0
$$

The plot is to show that (b) implies that $\Psi \equiv 0$, so that $\phi_{2}=0$. Hence every $L^{1}\left(\mathbf{R}_{+}\right)$function in $L^{\mathbf{1}}\left(\mathbf{R}_{+}^{2}, \mathbf{R}_{+}\right)$is in $I(f)$, and since every function in $L^{1}\left(\mathbf{R}_{+}^{2}\right)$ can be approximated by convolution products of a function in $L^{1}\left(\mathbf{R}_{+}\right)$and one in $L^{1}\left(\mathbf{R}_{+}^{2}\right)$, we conclude that $I(f)=L^{1}\left(\mathbf{R}_{+}^{2}, \mathbf{R}_{+}\right)$.

Let $L_{e}^{1}\left(\mathbf{R}_{+}\right)$be the unitization of $L^{1}\left(\mathbf{R}_{+}\right)$, and identify the unit with the Dirac measure at 0 , which we denote by $\delta_{0}$, too. Introduce the partial Fourier transform

$$
\mathcal{F}_{1} f\left(z_{1}, t_{2}\right)=\int_{0}^{\infty} f_{1}\left(t_{1}, t_{2}\right) e^{-i t_{1} z_{1}} d t_{1}+\delta_{0} \int_{0}^{\infty} f_{2}\left(t_{1}\right) e^{-i t_{1} z_{1}} d t_{1}, \quad t_{2} \in \mathbf{R}_{+},
$$

which is an element of $L_{e}^{1}\left(\mathbf{R}_{+}\right)$for fixed $z_{1} \in \bar{\Pi}_{-}$. As in the proofs of Theorems $2.1,3.1$, and 4.1 , the key to showing $\Psi \equiv 0$ is to get an estimate of $|\Psi|$ which is obtained by estimating the size of the norm $\left\|\left(\mathcal{F}_{1} f\left(z_{1}, \cdot\right)\right)^{*-1}\right\|_{L_{e}^{1}\left(\mathbf{R}_{+}\right)}$. In view of Proposition 1.2 and Lemma 1.3, we have good control of the sup-norm of the Fourier transform of $\left(\mathcal{F}_{1} f\left(z_{1}, \cdot\right)\right)^{*-1}$, which is $\sup _{z_{2} \in \bar{\Pi}} .1 /\left|\hat{f}\left(z_{1}, z_{2}\right)\right|$. In fact, along the ray $z_{1}=-i y, y>0$, the logarithm of this quantity is $o(y)$ as $y \rightarrow+\infty$, because on the Fourier transform side, (b) translates to say that (see [KOO, pp. 183-184])

$$
\lim _{y \rightarrow+\infty} y^{-1} \log \left|\hat{f}\left(-i y, z_{2}\right)\right|=0
$$

for all $z_{2} \in \bar{\Pi}_{-} \cup\{\infty\}$. The problem is how to convert this information into an estimate of the $L_{e}^{1}\left(\mathbf{R}_{+}\right)$norm. This is where condition (5.1) comes in: it implies that

$$
\sup _{z_{1} \in \Pi_{-}} \int_{0+}^{\infty}\left(1+t_{2}^{2}\right)\left|\mathcal{F}_{1} f\left(z_{1}, t_{2}\right)\right|^{2} d t_{2}<\infty
$$

Consider the space $L_{1}^{2}\left(\mathbf{R}_{+}\right)$of measurable functions $g$ on $\mathbf{R}_{+}$for which

$$
\int_{0}^{\infty}\left(1+x^{2}\right)|g(x)|^{2} d x<\infty
$$

clearly $L_{1}^{2}\left(\mathbf{R}_{+}\right) \subset L^{1}\left(\mathbf{R}_{+}\right)$. When equipped with convolution multiplication and the natural Hilbert space norm, $L_{1}^{2}\left(\mathbf{R}_{+}\right)$is a Banach algebra (see $[\mathbf{T C H}]$ ), and its image under the (injective) Gelfand (or Fourier) transform is the space $H_{1}^{2}\left(\Pi_{-}\right)$ 
consisting of those $g \in H^{2}\left(\Pi_{-}\right)$for which $g^{\prime} \in H^{2}\left(\Pi_{-}\right)$. Denote by $H_{1}^{2}\left(\Pi_{-}\right) \oplus \mathbf{C}$ the unitization of $H_{1}^{2}\left(\Pi_{-}\right)$. Assume $g=\varphi+\lambda \in H_{1}^{2}\left(\Pi_{-}\right) \oplus \mathbf{C}$ is invertible, with $\varphi \in H_{1}^{2}\left(\Pi_{-}\right)$and $\lambda \in \mathbf{C}$, and

$$
\|g\|_{H_{1}^{2}\left(\Pi_{-}\right) \oplus \mathrm{C}} \leq 1, \quad\|1 / g\|_{H^{\infty}\left(\Pi_{-}\right)} \leq C .
$$

What can then be said about the $H_{1}^{2}\left(\Pi_{-}\right) \oplus \mathbf{C}$-norm of $1 / g$ ? This is the question we need to resolve to convert the estimate of the sup-norm of the Fourier transform of $\left(\mathcal{F}_{1} f(z, \cdot)\right)^{*-1}$ into an estimate of the actual $L_{e}^{1}\left(\mathbf{R}_{+}\right)$-norm (observe that the norm on $L_{1}^{2}\left(\mathbf{R}_{+}\right)$is stronger than that of $\left.L^{1}\left(\mathbf{R}_{+}\right)\right)$. An estimate of $\|1 / g\|_{H_{1}^{2}\left(\Pi_{-}\right) \oplus \mathbf{C}}$ that grows like a power of $C$ will be perfectly acceptable, because then the fact that

$$
\sup _{z_{2} \in \Pi_{-}} \frac{\left|\operatorname{Im} z_{1}\right|}{\left|z_{1}\right|^{2}+1} \log 1 /\left|\hat{f}\left(z_{1}, z_{2}\right)\right|
$$

is uniformly bounded in $z_{1} \in \Pi_{-}$, which is a consequence of Lemma 1.3, or rather, its proof, will entail that

$$
\frac{\left|\operatorname{Im} z_{1}\right|}{\left|z_{1}\right|^{2}+1} \log \left\|\left(\mathcal{F}_{1} f\left(z_{1}, \cdot\right)\right)^{*-1}\right\|_{L_{e}^{1}\left(\mathbf{R}_{+}\right)}
$$

is uniformly bounded, and when one of these quantities tends to 0 as $z_{1} \rightarrow \infty$, the other one does, too.

Since $(1 / g)^{\prime}=-g^{\prime} /(g)^{2}$,

$$
\left\|(1 / g)^{\prime}\right\|_{H^{2}\left(\Pi_{-}\right)} \leq\left\|g^{\prime}\right\|_{H^{2}\left(\Pi_{-}\right)} \cdot\|1 / g\|_{H^{\infty}\left(\Pi_{-}\right)}^{2} \leq C^{2},
$$

so what remains to be shown is that we can control $\|1 / g-1 / \lambda\|_{H^{2}\left(\Pi_{-}\right)}+1 /|\lambda|$. Clearly, $1 / C \leq|\lambda| \leq 1$. Now

$$
\int_{\mathbf{R}}|1 / g(x)-1 / \lambda|^{2} d x=|\lambda|^{-2} \int_{\mathbf{R}}|\varphi(x)|^{2} \cdot|g(x)|^{-2} d x \leq C^{4} \int_{\mathbf{R}}|\varphi(x)|^{2} d x \leq C^{4},
$$

which enables us to conclude that the $H_{1}^{2}\left(\Pi_{-}\right) \oplus \mathbf{C}$-norm of $1 / g$ grows like the square of $C$.

The final touches on the proof - to show $\Psi \equiv 0-$ can be filled in by the reader by studying carefully the proofs of Theorems 2.1 and 4.1 and possibly consulting [NYM, GUR, or HED].

REMARK 5.2. (a) One is inclined to suspect that Theorem 5.1 is true also without condition (5.1).

(b) Some functions in $L^{1}\left(\mathbf{R}_{+}^{2}\right)$ are convolution products of a measure in $L^{1}\left(\mathbf{R}_{+}^{2}, \mathbf{R}_{+}\right)$and a finite Borel measure on $\left(\mathbf{R}_{+} \cup\{0\}\right) \times \mathbf{R}_{+}$that is absolutely continuous with respect to area measure on $\mathbf{R}_{+}^{2}$, and with respect to linear measure on $\{0\} \times \mathbf{R}_{+}$. Theorem 5.1 gives some insight about the solution to Levin's problem for such functions.

6. Final remarks. A natural question is what happens in $\S 2$ if we consider closures of finitely generated instead of principal ideals. Let $f_{1}, \ldots, f_{n} \in A\left(\mathbf{D}^{2}\right)$ have $\bigcap_{j} Z\left(f_{j}\right) \subset\{1\} \times \overline{\mathbf{D}}$. When does the closure $I\left(f_{1}, \ldots, f_{n}\right)$ of the $A\left(\mathbf{D}^{2}\right)$-ideal generated by $f_{1}, \ldots, f_{n}$ contain $I(\{1\} \times \overline{\mathbf{D}})$ ? It is not hard to see that the condition

$$
\inf _{z_{2} \in \mathbf{D}} \limsup _{t \rightarrow 1^{-}} \max _{j}(1-t) \log \left|f_{j}\left(t, z_{2}\right)\right|=0
$$


is necessary. On the other hand, it is not sufficient, because the ideal appearing in Example 2.5(b) is generated (after closure) by the functions

$$
f_{1}\left(z_{1}, z_{2}\right)=z_{1}-z_{2}, \quad\left(z_{1}, z_{2}\right) \in \mathbf{D}^{2}
$$

and

$$
f_{2}\left(z_{1}, z_{2}\right)=\left(z_{1}-1\right) \exp \left(\left(z_{1}+1\right) /\left(z_{1}-1\right)\right), \quad\left(z_{1}, z_{2}\right) \in \mathbf{D}^{2},
$$

and yet these functions meet (6.1).

However, one can show that $I\left(f_{1}, \ldots, f_{n}\right) \supset I(\{1\} \times \overline{\mathbf{D}})$ holds if

$$
\inf _{z_{2} \in \mathbf{D}} \max _{j} \log \left|f_{j}\left(z_{1}, z_{2}\right)\right| \geq-C /\left(1-\left|z_{1}\right|\right), \quad z_{1} \in \mathbf{D}
$$

for some constant $C$, and

$$
\lim _{t \rightarrow 1^{-}} \inf _{z_{2} \in \mathbf{D}} \max _{j}(1-t) \log \left|f_{j}\left(t, z_{2}\right)\right|=0 .
$$

To see how to obtain this result, fix $\varsigma \in \mathbf{D}$, and consider the "corona problem" of finding $g_{1}, \ldots, g_{n} \in A(\mathbf{D})$ such that

$$
\sum_{j=1}^{n} f_{j}(\varsigma, z) g_{j}(z)=1, \quad z \in \mathbf{D}
$$

Our assumptions (6.2)-(6.3) are in terms of control of the quantity

$$
\delta(\varsigma)=\inf _{z \in \mathbf{D}} \max _{j}\left|f_{j}(\varsigma, z)\right| .
$$

By Wolff's solution to the corona problem for $H^{\infty}$ (D) [GAR, p. 327], we can find $g_{1}, \ldots, g_{n} \in H^{\infty}(\mathbf{D})$ satisfying (6.4) such that

$$
\left\|g_{j}\right\|_{\infty} \leq C \cdot n^{2} \cdot \delta(\varsigma)^{-3}, \quad j=1, \ldots, n,
$$

for some constant $C$. To convert these corona solutions into disc algebra functions, we can use the following trick, which was communicated to me by Donald Marshall. Let $r \in(0,1)$ and observe that

$$
\sum_{j=1}^{n} f_{j}(\varsigma, r z) g_{j}(r z)=1, \quad z \in \mathbf{D} .
$$

If $r$ is sufficiently close to 1 ,

$$
\left\|\sum_{j=1}^{n} f_{j}(\zeta, z) g_{j}(r z)-1\right\|<\frac{1}{2}
$$

because $f_{j} \in A\left(\mathbf{D}^{2}\right)$. Then

$$
\tilde{g}_{j}(z)=g_{j}(r z) /\left(\sum_{j=1}^{n} f_{j}(\zeta, z) g(r z)\right), \quad z \in \mathbf{D},
$$

is in $A(\mathbf{D})$, solves (6.4), and satisfies (6.5), too, because $\left\|\tilde{g}_{j}\right\|_{\infty} \leq 2\left\|g_{j}\right\|$. Put

$$
f_{\varsigma}\left(z_{1}, z_{2}\right)=\sum_{j=1}^{n} f_{j}\left(z_{1}, z_{2}\right) \tilde{g}_{j}\left(z_{2}\right), \quad\left(z_{1}, z_{2}\right) \in \mathbf{D}^{2},
$$


which is an element of $I\left(f_{1}, \ldots, f_{n}\right)$, and satisfies $f_{\varsigma}\left(\varsigma, z_{2}\right)=1, z_{2} \in \mathbf{D}$, and

$$
\left\|f_{\varsigma}\right\| \leq C \cdot n^{2} \cdot \delta(\varsigma)^{-3} \sum_{j=1}^{n}\left\|f_{j}\right\|=C(n) \delta(\varsigma)^{-3}
$$

The function

$$
T_{\varsigma} f_{\varsigma}\left(z_{1}, z_{2}\right)=\left(f_{\varsigma}\left(z_{1}, z_{2}\right)-1\right) /\left(z_{1}-\varsigma\right), \quad\left(z_{1}, z_{2}\right) \in \mathbf{D}^{2},
$$

is an element of the coset $\left(\varsigma-z_{1}+I\left(f_{1}, \ldots, f_{n}\right)\right)^{-1}$ for $\zeta \in \mathbf{D}$, so by (6.6) our assumptions (6.2)-(6.3) on $f_{1}, \ldots, f_{n}$ will enable us to pull through the proof of Theorem 2.1. Unfortunately, neither (6.2) nor (6.3) is necessary, as the following example shows.

EXAMPLE 6.1. For $1 / 2<\alpha<1$, let $\varphi_{\alpha}$ be the disc algebra function

$$
\varphi_{\alpha}(z)=(z-1) \exp \left(-((1+z) /(1-z))^{\alpha}\right), \quad z \in \mathbf{D} .
$$

It is easy to check that $\varphi_{\alpha}$ is an outer function. Put

$$
f\left(z_{1}, z_{2}\right)=\varphi_{\alpha}\left(z_{2}\right) \quad \text { and } \quad g\left(z_{1}, z_{2}\right)=1-z_{2}-\left(1-z_{1}\right)^{2}, \quad\left(z_{1}, z_{2}\right) \in \mathbf{D}^{2} .
$$

By Theorem $2.1, I(f)=I(\overline{\mathbf{D}} \times\{1\})$, and since $g\left(z_{1}, 1\right)=\left(1-z_{1}\right)^{2}$ is outer and vanishes at $z_{1}=1$ only, we conclude that $I(f, g)=I(\{(1,1)\})$. On the other hand,

$$
\lim _{t \rightarrow 1^{-}} \inf _{z_{2} \in \mathbf{D}}(1-t) \log \max \left\{\left|f\left(t, z_{2}\right)\right|,\left|g\left(t, z_{2}\right)\right|\right\}=-\infty .
$$

7. Acknowledgments. The author wishes to thank Boris Korenblum and Donald Marshall for many interesting discussions.

\section{BIBLIOGRAPHY}

[ACD] O. P. Agrawal, D. N. Clark and R. G. Douglas, Invariant subspaces in the polydisk, Pacific J. Math. 121 (1986), 1-11.

[BEG] C. Bennett and J. E. Gilbert, Homogeneous algebras on the circle. I, Ann. Inst. Fourier (Grenoble) 22 (1972), 1-19.

[CAR] T. Carleman, Les fonctions quasi-analytiques, Gauthier-Villars, Paris, 1926.

[DOM] Y. Domar, On the analytic transform of bounded linear functionals on certain Banach algebras, Studia Math. 53 (1975), 203-224.

[GAM] T. W. Gamelin, Lectures on $H^{\infty}(D)$, Notas de Mathematica, No. 21, Universidad Nacional de La Plata, La Plata, Argentina, 1972.

[GAR] J. B. Garnett, Bounded analytic functions, Academic Press, New York, 1981.

[GRS] I. M. Gelfand, D. A. Ră̌kov and G. E. Silov, Commutative normed rings, Chelsea, Bronx, N.Y., 1964.

[GUR] V. P. Gurarǐ̌, Harmonic analysis in spaces with a weight, Trudy Mat. Obshch. 35 (1976), 21-76; English transl., Trans. Moscow Math. Soc. 35 (1979), 21-75.

[HED] H. Hedenmalm, On the primary ideal structure at infinity for analytic Beurling algebras, Ark. Mat. 23 (1985), 129-158.

[HOF] K. Hoffman, Banach spaces of analytic functions, Prentice-Hall, Englewood Cliffs, N.J., 1962.

[JAN] M. R. Janeba, Analytic structures in certain compactifications of the unit ball and polydisc in $\mathbf{C}^{2}$, Dissertation, Univ. of California, Santa Barbara, 1983.

[KOO] P. Koosis, Introduction to $H_{p}$ spaces, London Math. Soc. Lecture Notes 40 (1980).

[KOR1] B. I. Korenblum, Invariant subspaces of the shift operator in a weighted Hilbert space, Mat. Sb. 89 (1972), $111-138$.

[KOR2] _ C Closed ideals in the ring $A^{n}$, Functional Anal. Appl. 6 (1972), 203-214. 
[LEV] B. Ya. Levin, Translates of functions of two variables, Problem 7.20, Linear and Complex Analysis Problem Book, 199 Research Problems, Lecture Notes in Math., vol. 1043, SpringerVerlag, Berlin, Heidelberg, New York, and Tokyo, 1984, p. 421.

[NYM] B. Nyman, On the one-dimensional translation group and semigroup in certain function spaces, Thesis, Uppsala, 1950.

[ROS] J. P. Rosay, Ine équivalence au corona problème dans $\mathbf{C}^{n}$ et un problème d'ideal dans $H^{\infty}(D)$, J. Functional Anal. 7 (1971), 71-84.

[RUS] L. A. Rubel and A. L. Shields, The failure of interior-exterior factorization in the polydisc and the ball, Tôhoku Math. J. 24 (1972), 409-413.

[RUD1] W. Rudin, The closed ideals in an algebra of analytic functions, Canad. J. Math. 9 (1957), 426-434.

[RUD2] - Function theory in polydiscs, Benjamin, New York, 1969.

[RUD3] _ , Real and complex analysis, Tata McGraw-Hill, 1974.

[TAW] B. A. Taylor and D. L. Williams, Ideals in rings of analytic functions with smooth boundary values, Canad. J. Math. 22 (1970), 1266-1283.

[TCH] P. Tchamitchian, Généralisation des algèbres de Beurling, Ann. Inst. Fourier (Grenoble) 34 (1984), 151-168.

\section{Department of Mathematics, Uppsala University, S-75238 UpPSala, SWeden}

Current address: Department of Mathematics, Michigan State University, East Lansing, Michigan 48824 